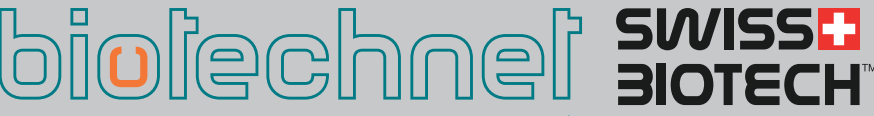

\section{BioTech 2019 - ZHAW Waedenswil, 2 - 3 July 2019: Part 1 From Innovation to Technology Breakthrough}

\section{Elsbeth Heinzelmann, Journalist Science and Technology}

When experts from all over the world met this year at ZHAW Waedenswil to discuss the possibilities of single-use technologies, the future had already arrived. Most of the developments have been implemented in upstream processing (USP), and complete single-use process platforms up to cubic metre scale are already a reality. This issue looks back on the 2019 BioTech conference, while the next will look at cellular agriculture, a technology that is ushering in a revolution in food production.

First let us allow Eric Langer, President and Managing Partner of US-based BioPlan Associates Inc., who has more than 20 years' experience in biotechnology and life sciences, to familiarise us with this future-oriented area.

\section{How single-use systems (SUS) are shaping the future of bioprocessing}

As Langer says, the world market for biopharmaceuticals is now worth over \$260 billion and is growing at a rate of approximately $12 \%$ CAGR (Compound Annual Growth Rate) over the past 10 or more years. New technologies such as cell and gene therapy, new products such as biosimilars, and new markets like China and India continue to sustain growth. As single-use biomanufacturing technology achieves maturity, it is supporting the associated increase in production by creating new and better options for future bioproduction. With a market share of $\geq 85 \%$ or more, single-use bioprocessing equipment now dominates the smaller pre-commercial bioprocessing market. The market for single-use equipment continues to nearly double every 5 years, and as new commercial-scale facilities come to be designed and built, they will incorporate the flexibility provided by single-use devices in combination with more modular facilities. Single-use systems are the obvious choice for emerging technologies such as cell therapy. However, very few purpose-built devices have as yet been developed and brought to market, which means that the field is still wide open. Thus, even though single-use technologies have been around for decades, innovation is just getting off the ground and opportunities are only now beginning to emerge. The shift towards single-use is being driven by bigger trends that include the demand for greater flexibility, more productivity and efficiency, cost containment and control, production in developing regions and the use of contract manufacturing organisations. The dominance of single-use bioprocessing equipment in clinical trial-scale manufacturing enables smaller and underfunded companies in particular to reduce the cost of facility construction. Furthermore, the technologies' rapid deployability and shorter time to run demonstrate their sustainability. The ability to merge single-use with modular technologies is already delivering greater process interconnectivity.

Single-use devices have become an enabling technology in all areas of bioprocessing, facilitating better, faster and more consistent processing at ever-larger scales. Nevertheless, some hurdles remain, including problems with integrity, particulates and data on leachables and extractables. This makes it essential to discuss the industry's view on a broad range of topics, from the need for greater standardisation to demand for single-use sensors and analytical devices.

www.bioplanassociates.com

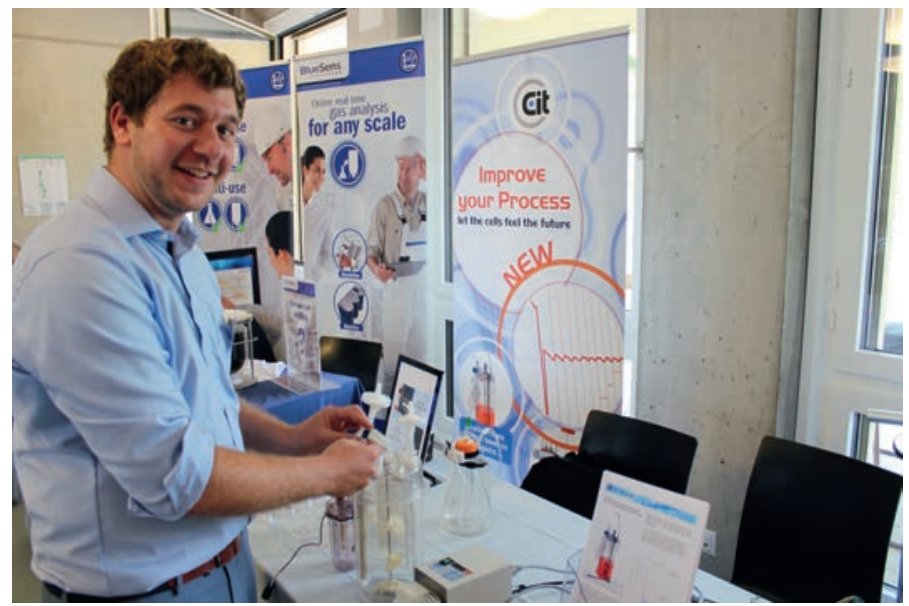

C-CIT Sensors offer the first process control system for bio-production based on in situ measurement of glucose and lactate, from smallest vessel to bioreactor scale. Contact: silvia.sturzenegger@c-cit.ch

\section{Intensifying the single-use process}

Stefan R. Schmidt is $\mathrm{COO}$ at BioAtrium AG, a joint venture between Lonza and Sanofi. He explained that traditional manufacturing processes for biologicals have been optimised over the last decade to deliver higher titers and better yields. "One of the multiple factors contributing to that improvement is process intensification", he commented. Process intensification involves using a wide range of strategies to combine, uncouple, streamline or eliminate unit operations with the aim of enhancing the productivity of manufacturing equipment and facilities. Many options exist that can be implemented at different points in the process. Interestingly a large number of these rely on disposable equipment.

For instance, reducing overall process duration by increasing cell densities became feasible when it was possible to freeze large-volume cell banks in bags or adopt Wave bags for the inoculum process. In downstream unit operation, steps in flow-through mode eliminate the need for intermediate tanks (virus inactivation in a tubular reactor, membrane chromatography, single pass TFF). Furthermore, combining process steps using a charged virus filter and mixed-mode resins reduces process time. The next step for integration and intensification are compact stand-alone instruments for delivering personalised cell therapy at the patient's bedside.

www.bioatrium.com

\section{The use of high-cell density cryopreservation}

Habib Horry is Associate Director of Marketing in charge of upstream solutions for next-generation processes at Merck KGaA in Darmstadt. He explained that seed train unit operation is the most time-consuming part of production in the majority 
of mammalian cell culture processes. The time requirement increases substantially as production scale grows, since additional expansion steps are required in response to increasing cell culture volumes in order to generate a sufficient amount of cell mass for the next scale-up step. While extended pre-culture expansion times are a key cause of process variability and increase the risk of contamination, process intensification strategies can help minimise cycle time, process variability and contamination risk.

Horry, who holds a $\mathrm{PhD}$ in microbiology and biotechnology from the University of Nantes, France, believes that high celldensity cryopreservation (HCDC) could play an important role in seed train process intensification by making it possible to freeze cells at high concentrations in specialised single-use bag assemblies. Using these frozen seed train intermediates could significantly reduce production cycle time, batch-to-batch variability and the risk of contamination. The seed train intermediates can be used as a single cell source at multiple stages in drug process development and manufacturing campaigns, thereby reducing turnover time and increasing flexibility.

Horry's team determined the optimum conditions in terms of cell densities, freeze and thaw temperatures, and dimethyl sulfoxide (DMSO) exposure for CHO (Chinese Hamster Ovary) cell lines used within the company. A high cell-density culture was generated in a perfused $\mathrm{N}-1$ bioreactor and decanted into customised single-use bag assemblies under different conditions. The frozen $\mathrm{N}-1$ seed train intermediates were then used to inoculate small-scale batch and perfusion bioreactors so that cell culture growth and productivity could be directly compared with a reference perfusion process in a standard seed train. www.merckgroup.com/de

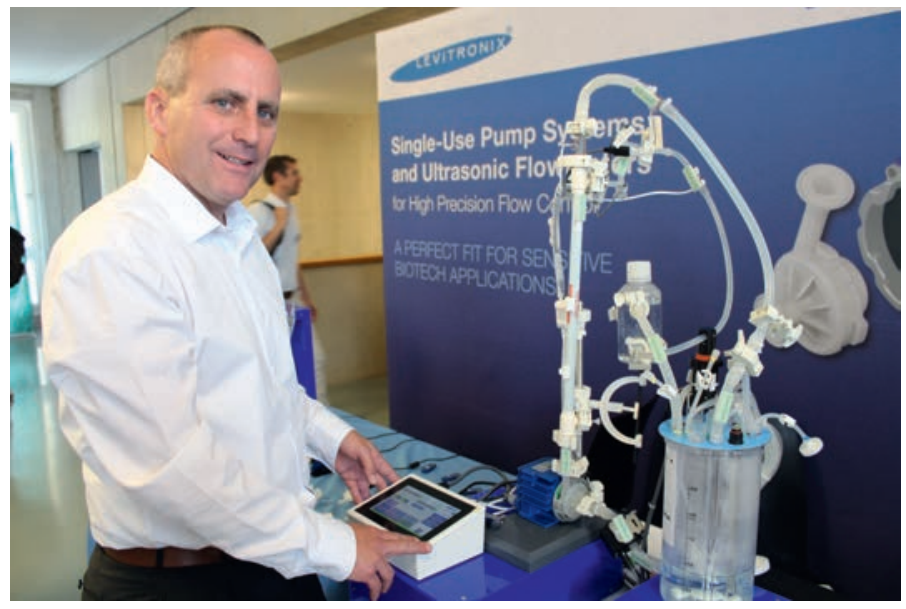

A Levitronix ${ }^{\circledR}$ disposable single-use pump head consists of only two pieces: the rotating impeller and the pump housing. During operation, the impeller levitates in the center of the pump housing, rotates, and at no time comes in contact with the static pump housing. The pump head is completely enclosed and there are no bearings to wear out or seals to breakdown. Contact: sibilia@levitronix.com

\section{Here come the new enhanced single-use bioreactors (SUBs)}

Nephi Jones is Associate Director Research and Process Development at Thermo Fisher Scientific in the USA. He is convinced that new application-specific bioprocessing tools, best practices and recommendations that leverage strategic enhancements to the sparger and agitation systems improve mixing \& mass transfer performance by more than $50 \%$ compared with legacy SUB designs. Data will include new system characterisation models and verification data showing the benefits of $50 \mathrm{~L}$, 100L, 250L and 500L SUBs, including extensive cell culture results. The reliability and consistency of the TruBio DeltaV system is further improved by the adoption of single-use conductivity-based foam probes (simple to implement and especially resistant to foam fouling effects). To address the unique needs of animal cell culture scale-up, these various enhancements have been fine tuned into three different application-specific SUB configurations. The optimisations have yielded superior results using CHO platforms of fed-batch and perfusion exceeding 260 million viable cells per $\mathrm{ml}$ using alternating tangential flow filtration (ATF) as well as African green monkey kidney (VERO) cells grown using high-concentration micro-carrier-adherent cultures. The end deliverable is a very flexible expansion of bioreactor capabilities. All end users of single-use bioreactors will want to be aware of these enhancements as a way of better meeting future demands for process intensification from the biotech and biomanufacturing industry.

www.thermofisher.com

\section{Digital technologies in modern bio-manufacturing}

William G. Whitford is Strategic Solutions Leader at GE Healthcare in the USA. He wanted to particularly highlight the role of digital technologies in contemporary bio-manufacturing. The key initiatives in the ongoing advancement of digital biomanufacturing include de-siloing data, predictive simulations, model reference adaptive control, process automation and dynamic business enterprise control algorithms. The collection, organisation and application of large amounts of data are major elements of these initiatives. One of the enabling technologies used here is big data, a term that generally refers to the use in statistical and mathematical algorithms of large amounts of structured and unstructured data from any source. Whitford, who has over 20 years' experience in biotechnology product and process development, pointed out that in bioprocessing, sources of data include new in-on-at-line monitoring from both product design and manufacturing. Academic activities such as published cellular platform metabolic pathways, flux and gene regulation, and general mammalian transcriptomic are another important source. GE is currently implementing AI (Artificial Intelligence) systems that will be able to read relevant publications incorporate new learnings into their process understanding and apply it in process development or control designs.

These data, coupled with well-designed algorithms and ultrafast computers, are providing both a quantitative understanding of cell physiology and advanced model-based control, enabling a new level of process development and operational efficiency. The ultimate goal is to generate an individualised metabolic network and process parameter models to represent specific entities, host cell lines and bioprocessing. Early digital twin learning and in silico models are now capable of creating simulations that can improve process development, control and event prediction. www.gehealthcare.com

\section{And now some regulatory considerations}

Daniel Müller is head of the regional GMP inspectorate at Regierungspraesidium Tuebingen, Baden-Württemberg, Germany. His area of expertise is active pharmaceutical ingredients (drug substances) and drug products manufactured by biotechnology or genetic engineering. He senses a lack of official regulatory guidance on single-use systems (SUS). As he commented, there are very few official guidance documents that deal with SUS, i.e., the plastic based components, assemblies and systems that are used in the production of drug substance or drug product, but do not form part of the final product packaging. Most official guidance documents or compendial monographs focus on primary packaging material for final drug products. While these documents may be helpful in defining requirements for single-use systems, such requirements have to be evaluated in the context of the production processes in which they are used. It is important 
to remember that product or process owners have to be able to justify using single-use systems in production processes. Thus, a GMP inspector will focus on the pharmaceutical company's risk analysis and rationale for employing plastic-based singleuse equipment. All these process aids - plastic components, assemblies or single-use systems - have to be of a sufficiently high and dependable quality to guarantee the quality of the drug substance or drug product. Since single-use equipment is generally sourced from external suppliers, thorough supplier qualification is mandatory for GMP-compliant production processes involving single-use equipment. gmp.inspector@gmx.de

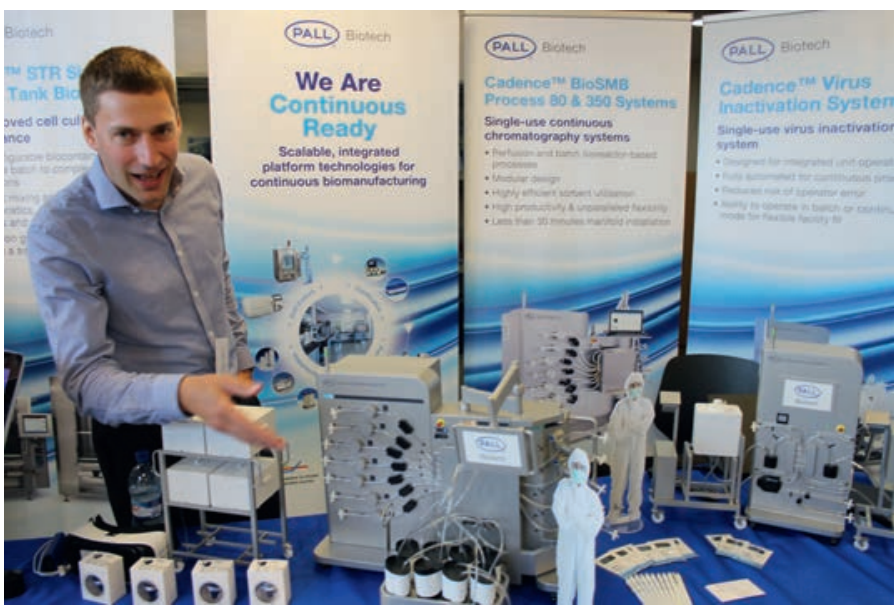

Pall's Cadence BioSMB platform is the first disposable flow path, continuous multi-column chromatography solution that is fully scalable from the Process Development (PD) laboratory to GMP manufacturing. Contact: valentin.ruettimann@pall.com

\section{Why a combination of unit operations and PAT?}

Detlef Eisenkrätzer is a Technology Expert at Roche Pharma Technical Development Europe in Penzberg. He postulated that a combination of unit operations and PAT (Process Analytical Technology) is necessary for intensified bioproduction. It is worth remembering that the majority of unit operations in the biopharmaceutical industry involve combining several unit operations in batch mode. Process intensification presents an opportunity to reduce both operating scale and the number of unit operations. When we take a closer look at process analytical technologies, we find that when a new unit operation is introduced to replace multiple batch unit operations, the new unit operation is usually more complex than the existing batch steps. This necessitates a higher level of control. The team at Penzberg evaluated the potential of different PAT technologies to control the new intensified unit operations. And what about continuous bioprocessing and harvesting technology? Apart from analytical technologies, new equipment is needed to replace the existing batch unit operation. Eisenkrätzer and his team compared devices for continuous cell culture and harvest steps to assess their potential for increasing the efficiency of the overall process.

For specific $\mathrm{CHO}$ processes, they selected the most suitable PAT methods for process control, the best equipment for continuous process steps and the leading process strategy. By implementing an intensified unit operation they were able to dramatically reduce process scale and operational costs.

https://www.roche.com/research_and_development.htm

\section{Single-use continuous downstream processing in} biopharma

Pall Biotech is the biopharmaceutical industry leader in single-use continuous downstream processing, and Britta Manser is its European manager in charge of scientific support for continuous bioprocessing. She talked about continuous bioprocessing as the next step in process intensification as downstream processing for biopharmaceuticals evolves. The perceived advantages of continuous manufacturing are its unprecedented control over product quality, greater agility and flexibility in responding to patient needs and reduced scale-up-related risks owing to smaller equipment and facilities. In addition, faster product release can facilitate a timely response to patient needs. There is clear potential for improving process cost-efficiency and product quality control, and the trend towards implementation of integrated processes by end users, suppliers and the regulatory community is visibly picking up speed and gaining significance.

Several adopters, including Bayer and Sanofi Aventis in Germany, and BMS, Merck and MedImmune in the USA, have shared successful examples of both fully integrated and hybrid processing. The first biosimilar monoclonal antibody produced using an integrated downstream process from BiosanaPharma in the Netherlands has been approved for phase 1 clinical trials, and results are expected at the end of the year.

Given the evident benefits for cost-efficiency and product quality control, it is claimed that there is now a need to address business challenges, technology gaps and regulatory uncertainties if continuous biomanufacturing is to gain widespread industry acceptance. https://biotech.pall.com/

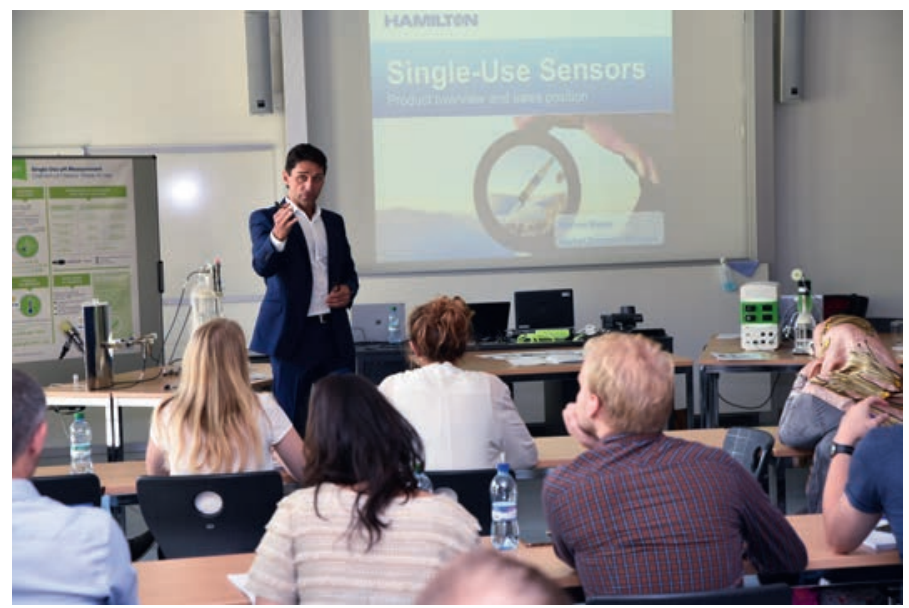

More measurement parameters and less measurement error yield tighter process control with connection to the tablet: this is new to HAMILTON! Contact: mbayer@hamilton.ch.

\section{Introducing the new $\mathbf{2 k L}$ hybrid single-use multi- product drug substance production facility}

Sven Kiesewetter is senior project manager and partner at Bideco AG in Uster. He talked about the new 2000 L hybrid single-use multiproduct drug substance manufacturing facility that F. Hoffmann-la Roche Ltd has built in Basel for the launch and commercial manufacturing of therapeutic proteins such as recombinant monoclonal antibodies. Designed to handle biosafety level 1 organisms, the facility has been installed on one floor of an existing building, and comprises a seed laboratory, media preparation, fermentation, harvesting, downstream processing and buffer storage area on a footprint of 1,200 $\mathrm{m}^{2}$. All product handling takes place in processes that are classified as closed or functionally closed under the 2013 ISPE Baseline Guide on Biopharmaceutical Manufacturing Facilities, with process equipment installed in ISO 9-classified rooms. There is no segregated personnel flow between upstream and downstream operations. Pre- and post-viral purification steps take place in the same shared room, as does API filling. The only ISO 8 environment is 
for seed laboratory activities, while buffer storage is housed in a controlled, but not classified, area. A decontamination autoclave has been installed for inactivation of any bio-contaminated solid wastes.

By providing utility stations in the different production rooms, the flexible and modular design approach makes connecting process equipment to utilities, energy sources and automation a quick and easy task, while also making it simpler to reconfigure equipment and respond to the demand for flexibility that multiproduct facilities have to fulfil. Single and multi-transfer ports have been installed in the cleanroom walls so that liquid media, product and buffers can be transferred between different rooms.

The 50 and $500 \mathrm{~L}$ cell expansion bioreactors and the 2000 $\mathrm{L}$ production bioreactors are designed as a super skid with one shared stainless-steel platform, which provides support to the pipe work of all utilities as well as for the cabling and single-use tubing relevant to the operation of the fermentation equipment. In addition, the platform provides safe and easy access to the upper part of the production bioreactors, where the ventilation filters and liquid inlets are located.

The overall project timeline from kick-off to handover (completed OQ) and start of the PPQ (Process Performance Qualification) campaign lasted 18 months.

www.bideco.com

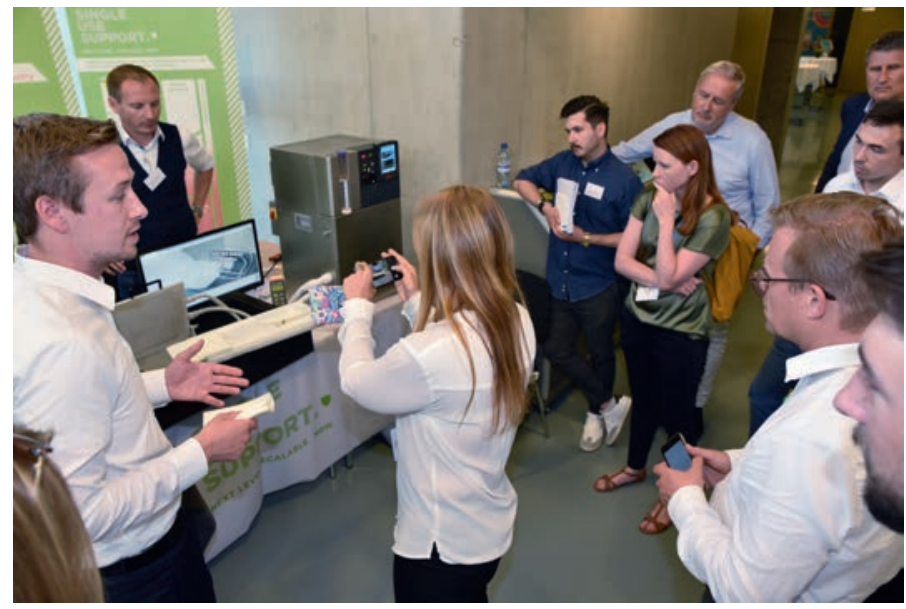

As Thomas Wurm, Managing Director and Co-Founder with Johannes Kirchmair of Single Use Support GmbH explain, RoSS ${ }^{\circledast}$ (robust storing and shipping) represents the first step toward $100 \%$ safety in liquid logistics.

\section{And finally, the scalable freeze-thaw platform}

Thomas Wurm, Managing Director and co-founder of Single Use Support GmbH, spoke about the company's single-use, bagindependent and scalable freeze-thaw platform for bulk drug substance. The main challenges of single-us bags right now are the scalability (same freezing and thawing kinetics with different batch sizes) and the brittleness and lack of robustness ab sub-zero temperatures. On the one hand, scaling up from clinical phases to market supply while retaining the same product quality is a challenge for the biopharma and cell and gene therapy industry. Drug substances, product and cells often have to be frozen to ensure product stability and quality, and single-use bottles or bags or multi-use stainless steel vessels are used to store them. Single-use options are preferred, but bottles are designed for open handling and limited in size and scalability. On the other hand, we have a dual source of single-use systems, which reduces dependency on a single supplier. Single-use bags are closed systems, but since they get brittle and sensitive at low temperatures, they are liable to split. Furthermore, there is no vendor-neutral freeze-thaw platform for bags.

However: there is a technology platform by single-use support, the smart design of the RoSS ${ }^{\circledR}$ system and the usage of a plate based controllable freeze-thaw units. RoSS ${ }^{\circledR}$ is a secondary packaging that immobilizes every single-use bag at frozen conditions to keep them protected. The top and bottom of the $\mathrm{RoSS}^{\circledR}$ are made of high-quality stainless steel, which offers various advantages. The first is maximum durability and strength. Secondly, when in direct contact with the bag, it provides both an ideal basis for effective initial and ongoing freezing and consistent and gentle defrosting or thawing. Thirdly, thanks to the design, tolerances and material strengths of the stainless-steel top and bottom, the $\operatorname{RoSS}^{\circledR}$ construction with developed inlays allows vibration. It also reduces structural tension within the single-use bag and acts as a shock absorber. In short, it is a single-use bag vendor-neutral independent platform.

www.susupport.com

\section{Unstoppable single-use technologies}

The message from the conference on single-use technologies in biopharmaceutical manufacturing and the symposium on cellular agriculture and single-use technologies held at ZHAW Waedenswil from 2 to 4 July 2019 is clear: single-use technologies are here to stay in biopharmaceutical production. As Professor Dieter Eibl, organiser and the scientific 'conscience' of the event puts it "The majority of today's developments have been implemented in upstream processing, where complete single-use process platforms up to cubic metre scale are already a reality. As companies set out to increase the efficiency of USP, process intensification solutions for inoculum production that are based on single-use perfusion technologies are now in demand." And Professor Regine Eibl points out that: "BioTech 2019 aimed to focus on the potential of and challenges associated with process intensification and continuous bioprocessing using single-use technologies in upstream and downstream processing and fill-and-finish in biopharmaceutical production. We address everybody interested in these developments." 